\title{
Interactive electron energy-loss elemental mapping by the "Imaging-Spectrum" method
}

\author{
Jean-Louis Lavergne $\left({ }^{1,2}\right)$, Jean-Michel Martin $\left({ }^{1}\right)$ and Michel Belin $\left({ }^{1}\right)$ \\ $\left({ }^{1}\right)$ Laboratoire de Tribologie et Dynamique des Systèmes, CNRS URA 855, Ecole Centrale de Lyon, \\ Département de Technologie des Surfaces, BP 163, 69131 Ecully, France \\ $\left({ }^{2}\right)$ KODAK-PATHE, Laboratoire d'Analyses, Z.I. Nord, 71102 Chalon-sur-Saone, France
}

(Received 25 November 1992, accepted 10 February 1993)

\begin{abstract}
Résumé. - Nous exposons le principe d'obtention d'images chimiques à l'aide d'une méthode permettant un dialogue interactif entre, d'une part l'image et d'autre part le spectre de pertes d'énergie électronique d'un échantillon ou d'une zone d'un échantillon. Cette méthode baptisée "ImageSpectre" utilise les possibilités offertes par la microscopie électronique à transmission filtrée en énergie (EFTEM) couplée avec un micro-ordinateur. Les expériences ont été réalisées avec un microscope ZEISS CEM 902 et un système de traitement d'images KONTRON. Un logiciel que nous avons développé sur micro-ordinateur permet de travailler après acquisition. Nous comparons cette technique avec la méthode Spectres-Image et évaluons l'avenir de la microscopie filtrée en énergie. Nous discutons de la résolution spectrale et spatiale de la méthode ainsi que de la dose nécessaire pour obtenir une série d'images par la méthode "Image-Spectre". Nous montrons l'intérêt d'une telle technique pour l'obtention d'une cartographie chimique à haute résolution spatiale.
\end{abstract}

\begin{abstract}
We have developed a method which performs chemical imaging with great interactivity between a selected filtered image and the EELS spectrum of a specimen or of a selected area of this specimen. This method, called "Imaging-Spectrum", uses the capabilities of the Energy Filtering Transmission Electron Microscopy (EFTEM) fitted with a personal computer. The experiments are performed with a ZEISS CEM 902 coupled with a KONTRON image analysis system. A software has been developed which allows off-line work with an IBM AT. Comparison is made between this method and the Spectrum-Imaging technique and the future of filtered imaging is discussed. Experiments were carried out to show the spectral and spatial resolution of this method and dose calculations were achieved. We demonstrate that such a technique is of interest for fast high resolution chemical mapping.
\end{abstract}

\section{Introduction.}

Electron Energy-Loss Spectroscopy (EELS) is a powerful tool for quantitative elemental analysis in materials sciences [1]. This tool gives the best analytical resolution in Transmission Electron Microscopy (TEM) [2]. For several years, high-resolution elemental mapping has been a subject of interest world-wide and the analytical tool selected by several laboratories to perform this 
mapping is TEM-EELS.

There are two ways of collecting electron energy-loss spectra: the serial method (one energy channel at a time) and the parallel method (several energy channels at the same time). EELS sensitivity has been greatly increased by using multi-channel arrays to detect in parallel the inelastically scattered electrons [3]. This type of detector is known as Parallel Electron Energy Loss Spectrometer (PEELS). Alternatively, a new tool has been developed to perform electron energy-loss imaging: the Energy-Filtering Transmission Electron Microscope (EFTEM). Several types of instrument have been developed. Microscopes having the energy dispersive filter either built in the optical path between objective and projector lenses [4] or as a post-column imaging filter behind [5] are commercially available and they are shown in figure 1.

Similarly to X-ray mapping using a Scanning Transmission Electron Microscope (STEM) coupled with an Energy Dispersive X-ray Spectrometer (EDXS), some laboratories are performing elemental mapping using STEM coupled with PEELS. The use of a PEELS coupled with a STEM was first introduced by Jeanguillaume and Colliex [7] as the "Spectrum-Imaging" method. Energy-filtered images are obtained from the collection of PEELS spectrum for each position of the STEM probe. Williams and Hunt [8] have developed a system around a VG HB 501 STEM which is able to store and later to process entire 1024 channels parallel EELS spectra including the associated dark-field and probe current signals at each picture element (pixel). The acquisition time for a $256 \times 256$ pixels image is about 2.4 hours ( $80 \mathrm{~ms}$ dwell-time for the core-loss spectrum and a dead time of $50 \mathrm{~ms}$ per pixel) [16] and it would not be uncommon that, during this time, the drift of the specimen could be up to $20 \mathrm{~nm}$. The storage capacity required by this technique is important and is in the range of 4 to 500 Mbytes with data compression. The post-processing time, which is independent of the acquisition time, may vary from a few minutes to several hours for a same $128 \times 128$ pixels image. Balossier and al [9] have developed a similar processing method based on a PHILIPS CM 30 STEM. They control the scanning coils with an external computer so as to automatically acquire several spectra with different energy shifts per pixel in order to build a multi-spectrum image and, by calculating first or second difference spectra for each pixel, to build a difference spectrum image. Leapman and Andrews [10] discuss the severe limitations of this technique when applied to biological or beam sensitive materials because of the drift and long acquisition times.

The aim of this paper is to introduce a new method in EELS elemental mapping. Using an EFTEM we have developed a method called "Imaging-Spectrum" which produces a portion of a core-loss EELS spectrum for any point of a given image with high speed and great interactivity between the spectrum, the image and the analyst. We will demonstrate how easy it is to obtain elemental maps rapidly with the Imaging-Spectrum approach. Some examples will be given and finally, we will present a dose comparison between Spectrum-Imaging and Imaging-Spectrum approaches.

\section{Experimental procedure.}

Electron Spectroscopic Imaging (ESI) is a general term. It includes the various operational imaging modes obtainable by selection of different energy-loss electrons arising from primary electron beam-specimen interaction [6]. The goal of Electron Spectroscopic Imaging (ESI) is to obtain an elemental map (or Z-contrast image) directly in a microscope. Reimer [11] has described all the imaging modes that can be used for ESI. Elemental mapping needs two or three filtered images below and beyond an edge of interest.

In order to collect energy-filtered images, we used a conventional TEM combined with a Castaing-Henry magnetic-sector / electrostatic-mirror (Zeiss CEM 902) [12-14]. The objective 
a)

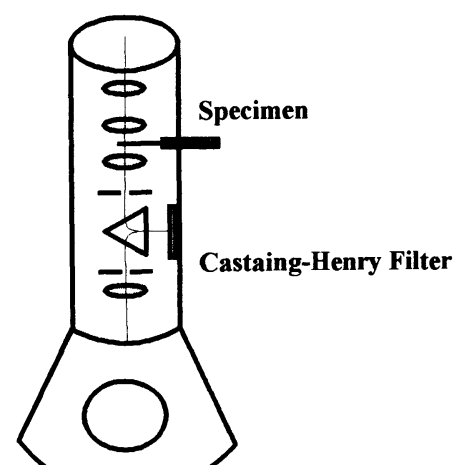

b)

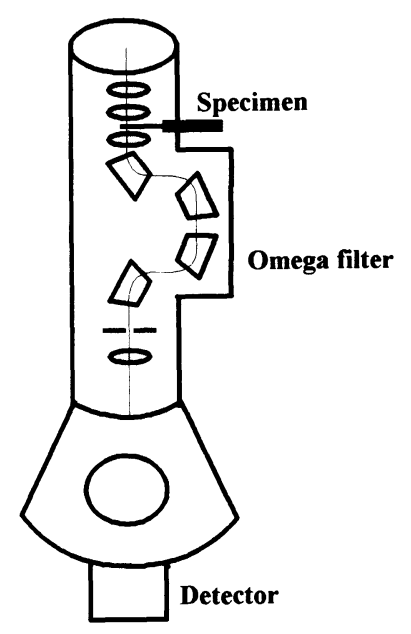

c)

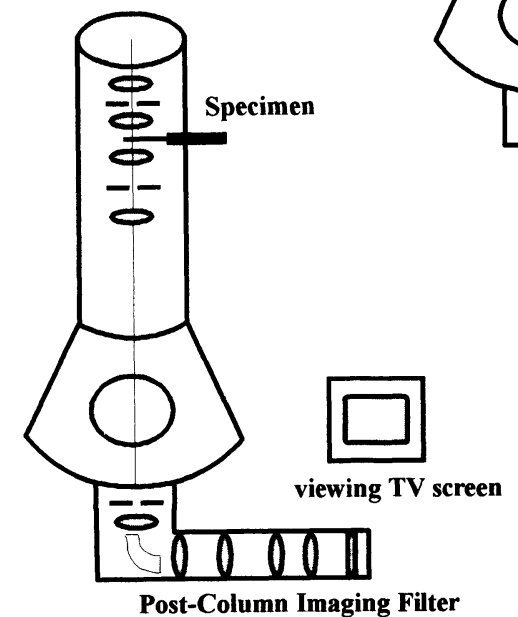

Fig. 1. - Types of imaging filters in energy-filtering TEM (EFTEM). a) Castaing-Henry filter, b) CastaingSenoussi or Omega filter, c) Post-Column Imaging filter.

aperture limits the scattering angle admitted to the filter. The lens placed after the filter projects either the achromatic image plane or the energy dispersive plane of the filter onto a detector (a camera for the image or a photomultiplier for the spectrum) [15]. These are the two modes of operating such a microscope. The image mode corresponds to a parallel acquisition of the filtered image and the spectrum mode allows serial recording of EELS spectra. If the microscope is connected to a computer all these acquisitions can be carried out automatically. 
In EELS elemental mapping, two kinds of information are under consideration:

- spatial dependence, which is provided by the variation of the signal from point to point on the specimen. Information $I$ is dependent on the two dimensions of the sample: $x$ and $y$,

- the chemical information, which stems from the spectroscopic response of the volume of material under analysis. Information $I$ is dependent on the energy: $E$.

Consequently, the complete data will be a three dimensional array: $I(x, y, E)$, as shown in figure 2 .

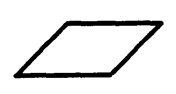

Conventional TEM image :

The image plane has no energy-loss information.

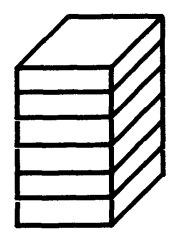

Imaging-Spectrum images :

Each image plane includes the value of the electron energy-loss selected by the energy selecting slit.

Several images are needed to have one whole spectrum per pixel.

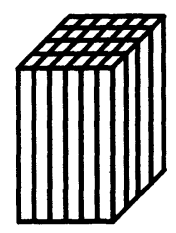

Spectrum-Imaging image :

Each pixel includes a complete spectrum. The image is reconstructed from a series of several spectra.

Fig. 2. - Types of images obtainable in a) CTEM, b) EFTEM + Imaging-Spectrum method and c) STEM + Spectrum-Imaging method.

The first method for the acquisition and processing of this three dimensional array is SpectrumImaging. A spectrum-image is similar to a normal image but it contains an entire PEELS spectrum at each pixel whereas an image plane has only a single value at each pixel. The Spectrum-Imaging method has been experimentally investigated by Hunt and Williams [16] and by Balossier et al [9].

The Imaging-Spectrum method is based upon the exploitation of a series of energy-filtered images, recorded at different energies with a chosen increment between each image (Fig. 3). Once the series of filtered images is recorded, the spectral information is extracted by reading the grey level value of a specific pixel over the total recording. The advantages of using an EFTEM is the parallel nature of the image formation. Data from a very large number of pixels (up to $1024 \times 1024$ ) can be acquired simultaneously.

The Imaging-Spectrum method was implemented on a ZEISS CEM 902. We wrote some specific software in $\mathrm{C}$ programming language for the acquisition. The instrument is equipped with a tungsten filament and a low level TV rate camera whose characteristics are described in table I. Implementation is presented in figure 4. The ZEISS CEM 902 is fitted with a built-in image analysis system. The system is designed around a KONTRON IBAS personal computer AT with an Intel 80286/287 microprocessor running at $12 \mathrm{MHz}$. The specific hardware of the system is a 4 Megabyte video memory (HDM), a MIAP 2 interface wich allows rapid numerical calculation, a MAC 3 acquisition card and an RTV video card. Mass-storage is achieved by a $40 \mathrm{Mb}$ hard disk. 


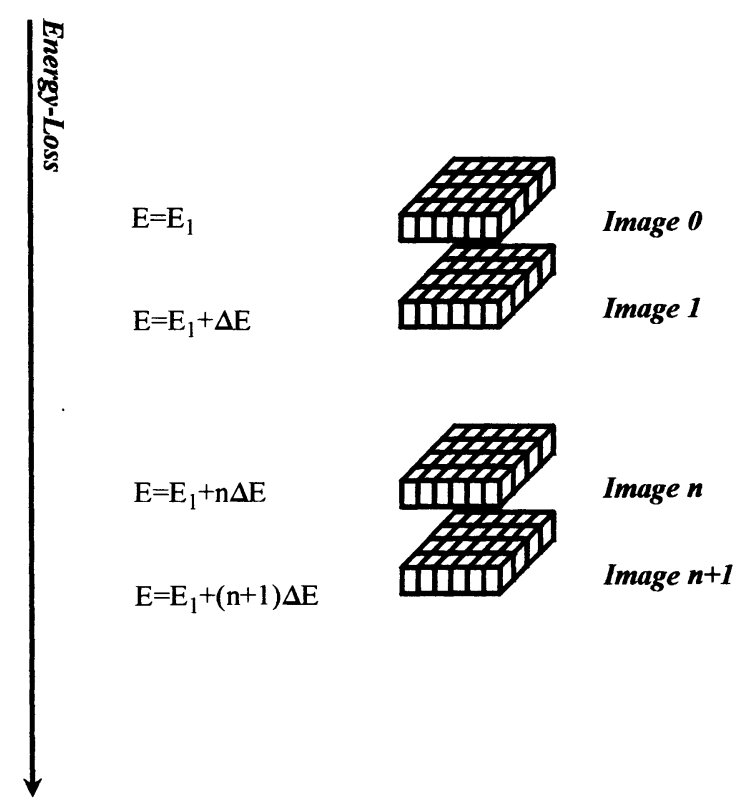

Fig. 3. - Principles of the Imaging-Spectrum method. The spectrum for a given pixel is obtained by reading the pixel's grey level value for each image.

This PC can drive both the EELS spectrometer and the image acquisition. The off-line processing is done with a PC AT 386/387 running at $25 \mathrm{MHz}$ fitted with a $110 \mathrm{Mb}$ hard disk and connected with the microscope through a local network. All data is stored on a $300 \mathrm{Mb}$ magneto-optical disk connected to the network. The off-line processing software is developed under Microsoft Windows interface. This configuration was chosen as it can be developed at a reasonable cost.

Table I. - Characteristics of the low level TV rate camera SIT model 66.

\begin{tabular}{|c|c|c|c|c|}
\hline $\begin{array}{c}\text { Minimum } \\
\text { illumination level }\end{array}$ & Resolution & $\begin{array}{c}\text { Amplification } \\
\text { input - output }\end{array}$ & Distortion & Dynamic range \\
\hline $10^{-7}$ lux & $\begin{array}{c}550 \text { lines center } \\
350 \text { corners }\end{array}$ & greater than $10^{9}$ & $\begin{array}{c}\text { Geometric }: 3 \% \\
\text { inearity }: 1 \%\end{array}$ & $256: 1$ \\
\hline
\end{tabular}

The acquisition of a series of $96256 \times 256$ pixels images takes no longer than for $961024 \times 1024$ images and depends on the signal under investigation. The processing of such a series is performed either by the image analysis system or by an off-line computer. The software which is written in Turbo-Pascal and developed in our laboratory allows, by a simple click with a mouse button, the display of an EELS spectrum corresponding to the selected pixel. This EELS spectrum is plotted with an energy step corresponding to the energy interval between each image: the number of points is the number of images. We have the possibility to select a single pixel, several pixels or a whole area depending on the counting statistics desired. The number of images 


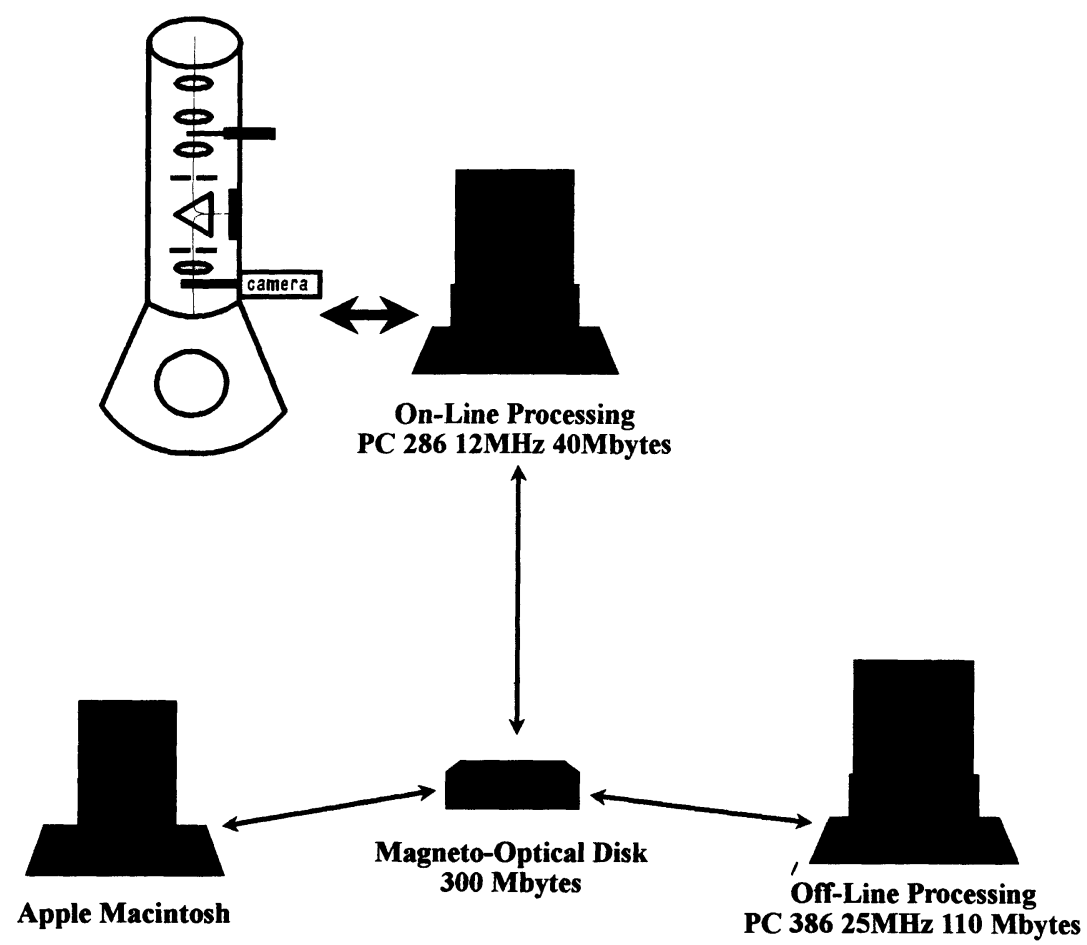

Fig. 4. - Schematic diagram of the Imaging-Spectrum configuration and the local network attached.

is limited by the storage capacity of the hard disk. The images are acquired with a 8 bit processor that gives a total amount of $6.3 \mathrm{Mbytes}$ for $256 \times 256$ pixels, $25 \mathrm{Mb}$ for $512 \times 512$ and $101 \mathrm{Mb}$ for $1024 \times 1024$ without any data compression.

The spatial resolution in the image is first governed by the normal aberration coefficients of the microscope. With a $C s=2.2 \mathrm{~mm}$ at $80 \mathrm{kV}$ acceleration voltage, the point resolution is 0.5 $\mathrm{nm}$. Considering the chromatic aberration, the energy window is a determinant factor and the resolution is approximately $0.6 \mathrm{~nm}$ for a $3 \mathrm{eV}$ selecting slit $(C c=1.7 \mathrm{~mm})$. It can be concluded that the resolution in the filtered image is practically conserved to a point of $1 \mathrm{~nm}$. The size of the analysed area is controlled by the choice of the image size and magnification, as shown in table II.

\section{Results and discussion.}

To illustrate the capabilities of the Imaging-Spectrum method, we conducted selected experiments on the ZEISS CEM 902 and the processing was carried out off-line with a PC. Table III summaries the operating conditions. The first specimen is a BN powder, with sub-micrometer grains. The preparation consists in depositing a droplet of a $\mathrm{BN}$ dispersion in ethanol, onto a holey carbon grid. Series of 96 images were recorded, with an energy step of $1 \mathrm{eV}$ (the energy interval scanned is 152 to $248 \mathrm{eV}$ ). The acquisition time for a single image was $3 \mathrm{sec}$. and the total acquisition time for a complete series (including the disk accesses) was 6 minutes. Figure 5a shows an energyfiltered image extracted from a series and corresponding to an energy loss of $163 \mathrm{eV}$ recorded with an energy selecting slit of $\delta E=3 \mathrm{eV}$ width. Several spectra could be obtained from a single 
Table II. - Correspondance between the magnification of the microscope (the image on the computer screen is 4 times bigger) and the minimum pixel size obtainable with the Imaging-Spectrum method (within the limits of the microscope resolution).

\begin{tabular}{|c|c|c|c|c|}
\hline $\begin{array}{c}\text { Image Size } \\
\text { (pixels) }\end{array}$ & $\times 200.000$ & $\times 100.000$ & $\times 30.000$ & $\times 20.000$ \\
\hline $256 \times 256$ & $0.75 \mathrm{~nm}$ & $1.5 \mathrm{~nm}$ & $5 \mathrm{~nm}$ & $7.5 \mathrm{~nm}$ \\
\hline $512 \times 512$ & $0.37 \mathrm{~nm}$ & $0.75 \mathrm{~nm}$ & $2.5 \mathrm{~nm}$ & $3.75 \mathrm{~nm}$ \\
\hline $1024 \times 1024$ & $0.19 \mathrm{~nm}$ & $0.37 \mathrm{~nm}$ & $1.25 \mathrm{~nm}$ & $1.9 \mathrm{~nm}$ \\
\hline
\end{tabular}

pixel (label 1,2 and 3 on this image) and are displayed in figure 6. Although the analysed area is as small as $25 \mathrm{~nm}^{2}$, the fine structure of the K-edge of boron is quite well resolved. The chemical mapping shown in figure 5b was obtained by a three image subtraction method $(163,183$ and 192 $\mathrm{eV}$ ) according to Egerton [17]. Following this way, it should be also possible to obtain $\sigma^{*}$ and $\pi^{*}$ orbital images, which could be of great interest for fine structure mapping.

Table III. - Operating conditions for the acquisition of the series of images for BN and Carbon (graphitised and amorphous).

\begin{tabular}{|c|c|c|c|c|}
\hline Sample & $E$ & Magnification & $\begin{array}{c}\text { Condenser } \\
\text { aperture }\end{array}$ & Intensity \\
\hline BN & $80 \mathrm{kV}$ & 30.000 & $90 \mu \mathrm{m}$ & $10^{-8} \mathrm{~A}$ \\
\hline Carbon & $80 \mathrm{kV}$ & 20.000 & $90 \mu \mathrm{m}$ & $10^{-8} \mathrm{~A}$ \\
\hline
\end{tabular}

In the second experiment, Highly Oriented PyroGraphite (HOPG) particles were deposited on a holey amorphous carbon grid. The sequence of 96 images between 236 and $332 \mathrm{eV}$ (carbon $\mathrm{K}$-edge spectrum) with an energy step of $1 \mathrm{eV}$ is recorded. The acquisition time per image is 5 sec. and the total acquisition time is 9.2 minutes. The selected magnification $(20,000)$ allows a spatial resolution of $7.5 \mathrm{~nm}$ per pixel. In order to obtain better counting statistics, we have selected the mean value of 5 pixels as a cross (shaped like $x$ ). Results show the real interactivity of the Imaging-Spectrum method in differentiating very quickly the fine structures of two kinds of carbon in the same specimen.

The imaging-Spectrum method can be favourably compared with a conventional serial EELS spectrum obtained with the EM902 in the diffraction coupling mode, with a selected area of $70 \times 10^{3}$ $\mathrm{nm}^{2}$. Figure 7 compares the two methods. Clearly, the spectral information obtained with the Imaging-Spectrum method seems to be valid enough to provide qualitative and quantitative information. It is important to note that the spectrum obtained from Imaging-Spectrum concerns a small area equivalent to $280 \mathrm{~nm}^{2}$ (the mean value of 5 pixels of $56 \mathrm{~nm}^{2}$ ) compared to $70 \times 10^{3}$ $\mathrm{nm}^{2}$ for the serial one. 

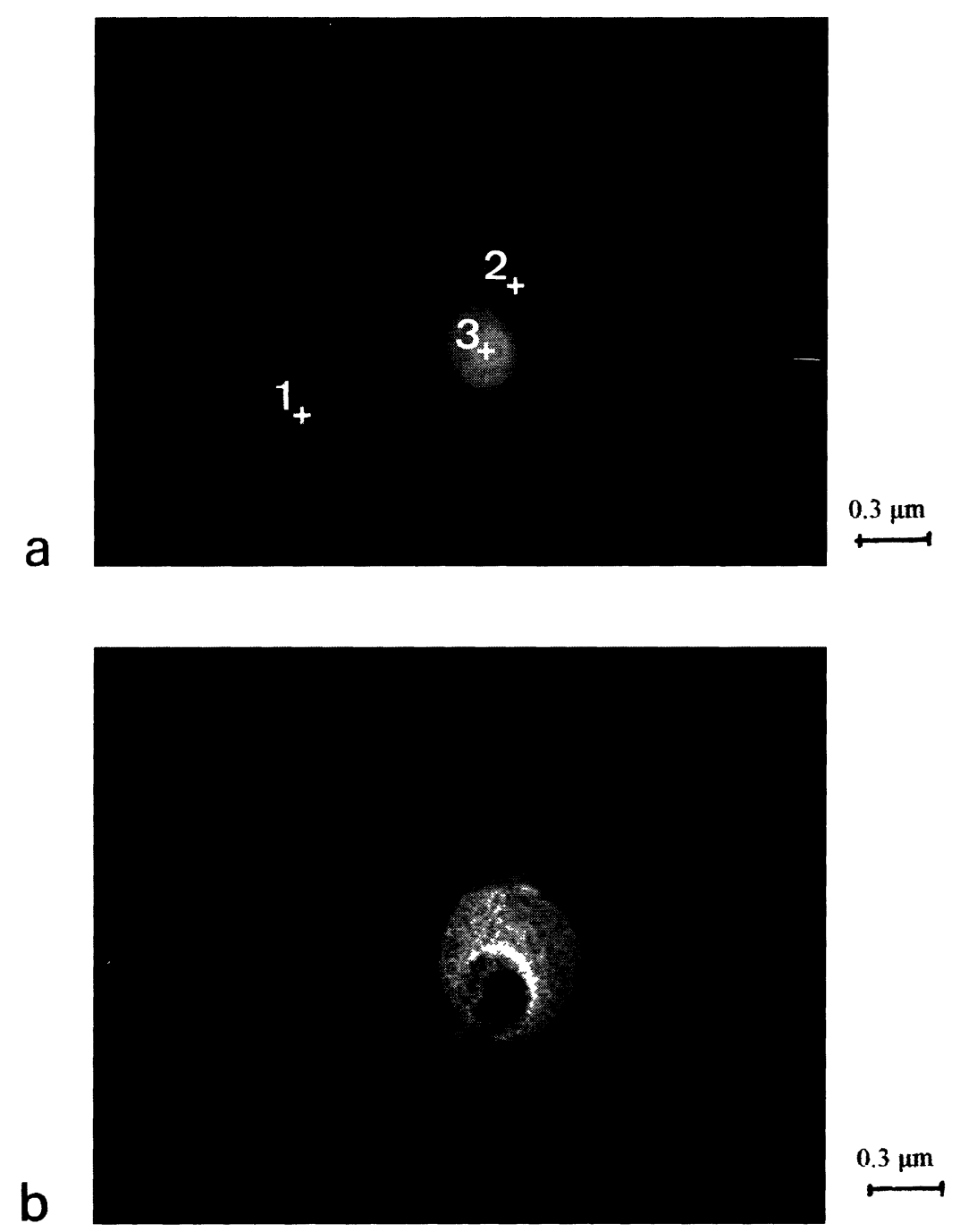

Fig. 5. - a) Filtered image ( $E=163 \mathrm{eV}, \delta E=3 \mathrm{eV}$, scattering angle $=17 \mathrm{mrad}$ ) of a particle of hexagonal boron nitride. The marked points are the analysed points for the spectra shown in Fig. 6; b) chemical image of the $\pi^{*}$ transition of boron in BN obtained by subtraction of 3 filtered images selected by the ImagingSpectrum method $\left(I_{1}=163 \mathrm{eV}, I_{2}=183 \mathrm{eV}\right.$ and $\left.I_{3}=192 \mathrm{eV}\right)$. The reversal of contrast in area 3 is due to a thickness effect.

Another important point to consider is the total electron dose arriving on the specimen during the acquisition phase. The dose in the ZEISS CEM902 is given by the relation:

$$
D_{\mathrm{s}}=\text { d.t. } M^{2}
$$

where $D_{\mathrm{s}}$ is the number of electrons achieved per specimen area $\left(\mathrm{e}^{-} \AA^{-2}\right), t$ is the exposure time (sec.), and $M$ is the magnification.

$d$ is a function of the detector size:

$$
d=\frac{i}{A . k . e}
$$




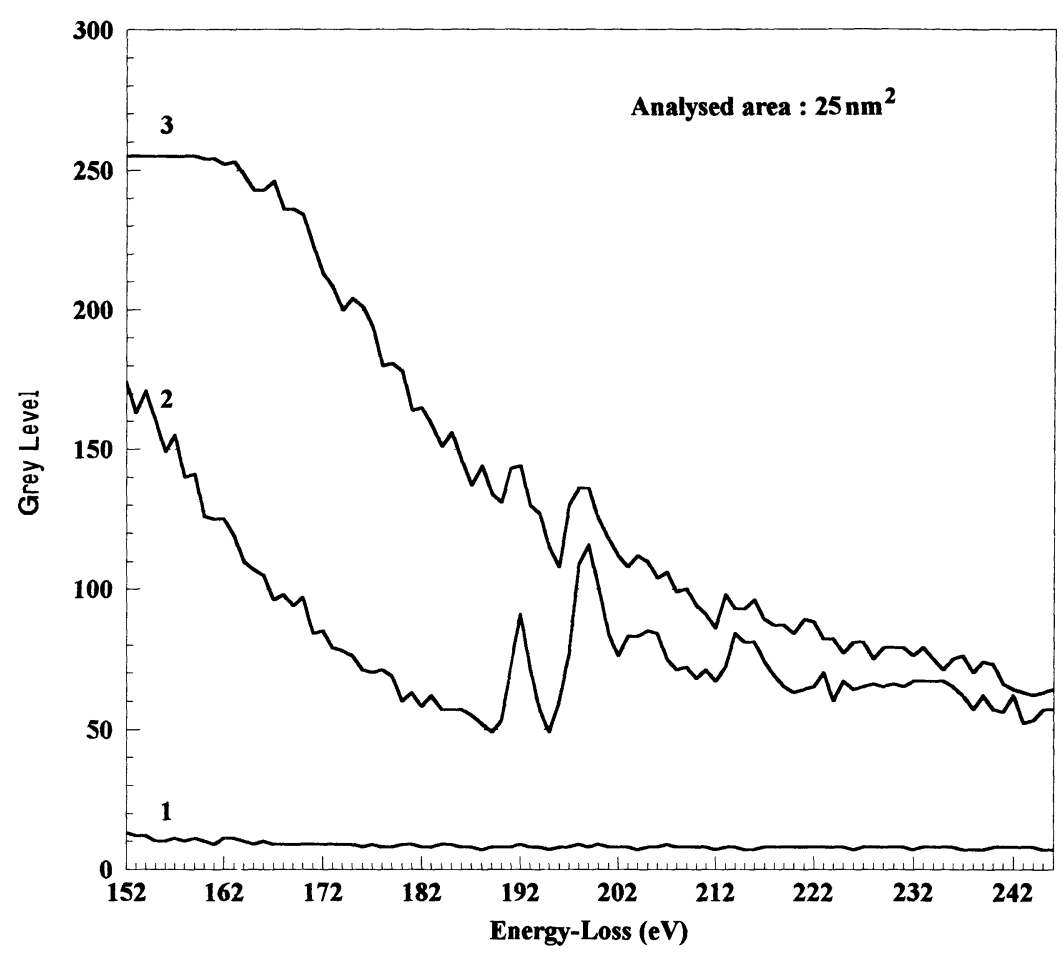

Fig. 6. - Unsmoothed spectra of the K edge of boron in hexagonal boron nitride obtained from 96 images with the Imaging-Spectrum method. The label on each spectrum refers to the corresponding area in figure 5 . The analysed area represented by a single pixel is $25 \mathrm{~nm}^{2}$.

where $i$ is the current intensity (A) measured from the camera detector with a nearly uniform illumination of the whole detector area with objective aperture out, $A$ is the detector area: $5.92 \times 10^{17}$ $\AA^{2}, k$ is a correction value which takes in consideration the ratio of backscattered electrons $(k=0.75), e$ is the charge of the electron $=1.602 \times 10^{-19} \mathrm{~A} \mathrm{sec}$.

In the case of the $\mathrm{BN}$ specimen, the current is equal to $5 \times 10^{-7} \mathrm{~A}$, the magnification is 30,000 and the acquisition time per series is $360 \mathrm{sec}$. The total dose achieved by the specimen during registration of the whole series is thus equal to $2.3 \times 10^{6} \mathrm{e}^{-} \AA^{-2}$. According to Leapman and Hunt [18], the probe diameter is $1 \mathrm{~nm}$. This gives a dose equal to $1.2 \times 10^{8} \mathrm{e}^{-} \AA^{-2} \mathrm{sec}^{-1}$. In the example given by Williams and Hunt [16], the acquisition time for a pixel is $80 \mathrm{~ms}$, giving a total dose equal to $9.6 \times 10^{6} \mathrm{e}^{-} \AA^{-2}$ for a whole image.

In the case of a thin carbon film $\left(\sigma_{\mathrm{K}}=2 \times 10^{-20} \mathrm{~cm}^{2}\right.$ at $\left.100 \mathrm{keV}\right)$, with a current density of $1 \mathrm{nA}$ $\mathrm{nm}^{-2}$, for a VG microscope, the count at each pixel on the carbon $\mathrm{K}$ edge is 12500 counts per second (cps). In our case the same calculation $\left(\sigma_{\mathrm{K}}=2.5 \times 10^{-20} \mathrm{~cm}^{2}\right.$ at $\left.80 \mathrm{keV}\right)$ with a current density of $7 \times 10^{-5} \mathrm{nA} \mathrm{nm}^{-2}$ gives $1 \mathrm{cps}$. This difference can be understood since the ZEISS is fitted with a tungsten filament and the VG has a field emission gun. This calculation shows that the acquisition time must be accorded with the cross-section of the element under consideration. We have to conduct experiments with another filament and to compare these results.

A normal high-resolution image generally requires $10^{2}$ to $10^{4} \mathrm{e}^{-} \AA^{-2}$. Both Imaging-Spectrum and Spectrum-Imaging require a higher dose with a slight advantage to Imaging-Spectrum because of its capabilities to reduce acquisition time without reducing the image size. 
a)

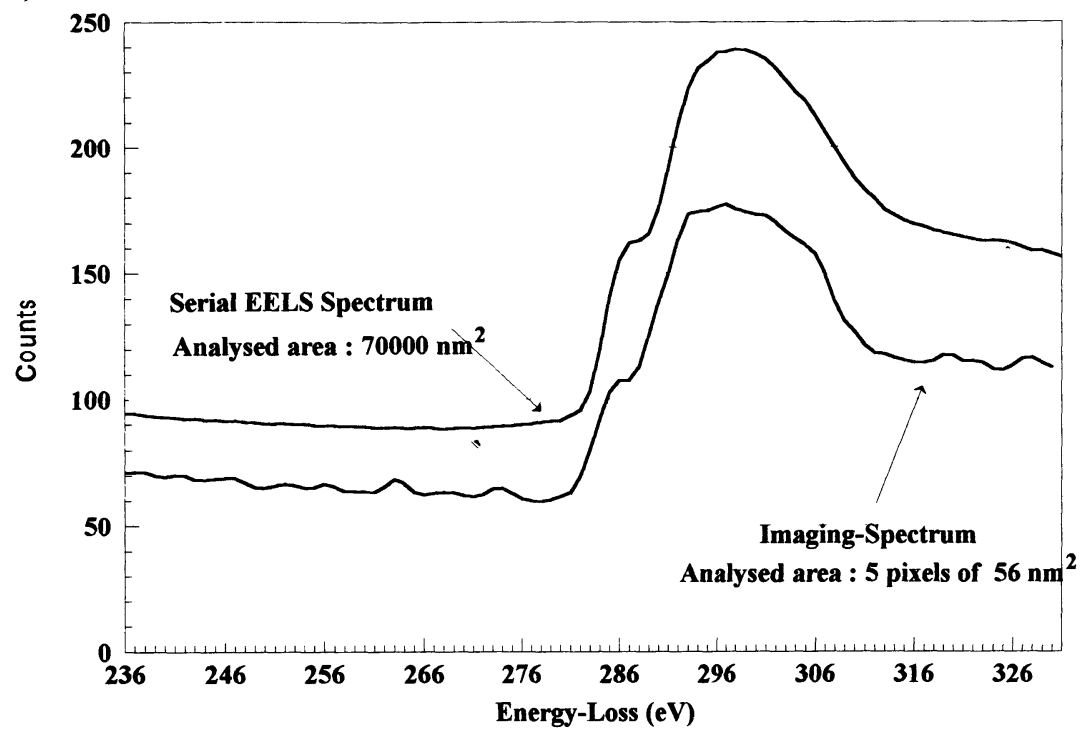

b)

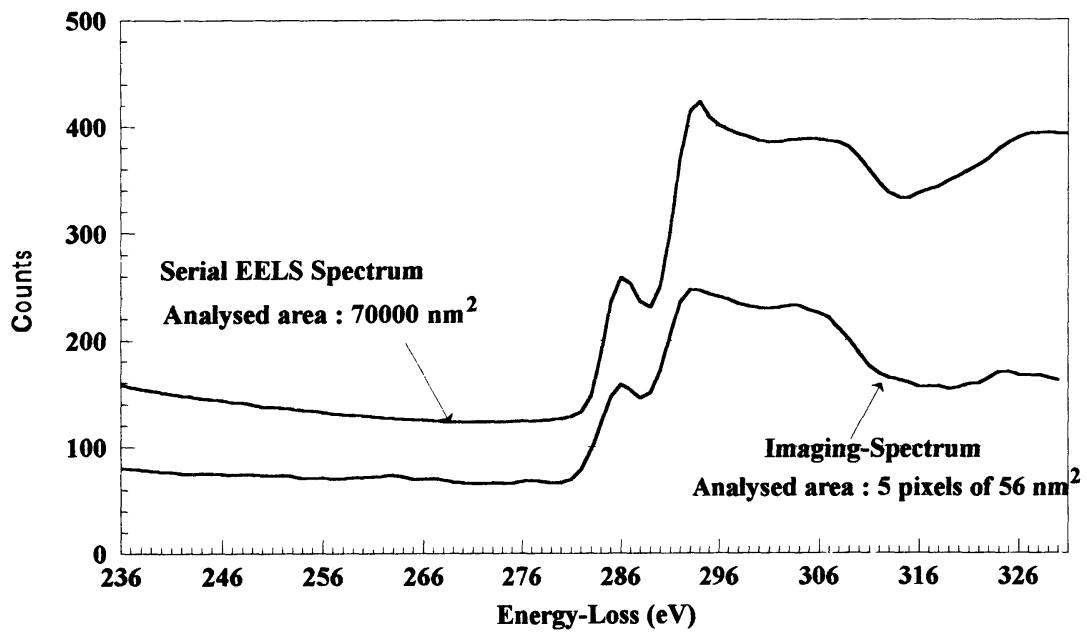

Fig. 7. - Comparison between serial EELS (analysed area $70.10^{3} \mathrm{~nm}^{2}$ ) and Imaging-Spectrum method (analysed area: mean value of 5 pixels of $56 \mathrm{~nm}^{2}$ ) for two samples: a) amorphous carbon and b) Highly Oriented PyroGraphite. Experiments were made on a ZEISS EM902, $80 \mathrm{kV}$.

Several parameters however limit the capabilities of the Imaging-Spectrum technique used in the present configuration. These are due to the microscope, the camera and the computer.

a) limitations due to the microscope:

- the ZEISS CEM902, when using the filter mode, introduces an energy dispersion up to $5 \mathrm{eV}$ between the corners and the centre of the image (due to the non-corrected second order aberrations),

- the energy selection is made by a mechanically controlled slit which is not accurate,

- the minimum energy step cannot be lower than $1 \mathrm{eV}$. 
b) limitations due to the camera:

- the DAGE SIT 66 has very good low level detection but introduces a variation of intensity in the image. This effect can be reduced by using a top-hat or a high-pass filter,

- the dynamic range of a TV rate camera is low (256:1) compared with a CCD (4096:1),

c) limitations due to the computer:

- the mass storage capacity of the on-line computer is only 40 MBytes which is inconvenient for multiple acquisitions,

- the video memory is too small and limits to 48 the number of images that can be stored simultaneously in memory ( $256 \times 256$ pixels images).

All these limitations can be easily reduced. For example by using a faster computer it will be possible to correct the non linearity of the camera in real-time and to increase the number of images or the resolution by simple algorithms. With the SIT camera, it is not possible to obtain spectra in the low-loss region, but with a camera whose dynamic range is higher this limitation can be eliminated. The procedure for correction of the energy dispersion in an image is to re-calibrate the spectrum for each pixel, which could be done off-line.

\section{Conclusion.}

Using an Energy-Filtering Transmission Electron Microscope (EFTEM), we have developed a method (Imaging-Spectrum) which acquires both filtered images and EELS spectra during a single experiment with great interactivity. It consists in recording a series of filtered images incremented in energy loss. With the equipement at hand, the method has demonstrated the following performance:

- the total acquisition time is no longer than five minutes and the overall dose $\left(10^{5} \mathrm{e}^{-} \AA^{2-}\right)$ similar to TEM experiments, which is particularly well adapted for moderately beam sensitive materials,

- from the data, a portion of a core-loss EELS spectrum (typically $96 \mathrm{eV}$ ) is immediately available for each image pixel. The spatial resolution of the EELS spectrum is very good, a carbon K-edge spectrum from an area smaller than $60 \mathrm{~nm}^{2}$ has been easily obtained on a graphite sample,

- the energy resolution in the EELS spectra has been checked by differenciating $\pi^{*}$ and $\sigma^{*}$ transitions in hexagonal $\mathrm{BN}$, indicating that a $2 \mathrm{eV}$ energy resolution has been achieved,

- fast chemical mapping with the same high spatial resolution is also possible. It is important to notice that the contrast on the chemical image can be compared to the EELS spectrum, at any time.

The future of the energy-filtered microscopy is more and more devoted to the obtention of images with quantitative spectroscopic information. The Spectrum-Imaging method allows the collection of high-quality 1024 channel energy-loss spectra on a small number of pixels. The Imaging-Spectrum method will enable the simultaneous and rapid collection of a high number of EELS spectra portions for a large number of pixels.

\section{Acknowledgements.}

The authors wish to thank J.-M. Hector, C. Gimenez and Y. Parent from KODAK-PATHE for their financial support and L. Carpentier from LTDS, for his help in the conversion of images files. 


\section{References}

[1] COLLIEX C., Microsc. Microanal. Microstruct. 2 (1991) 403-411.

[2] COLLIEX C., Microsc. Spectrosc. Electron. 7 (1982) 525-542.

[3] KRIVANEK O.L., AHN C.C. and KEENEY R.B., Ultramicroscopy 22 (1987) 103-116.

[4] FROMM I., REIMER L. and RENNEKAMP R., J. Microsc. 166 (1992) 257-271.

[5] KRIVANEK O.L., GUBBENS A.J. and DELLBY N., Microsc. Microanal. Microstruct. 2 (1991) $315-$ 332.

[6 TETLEY L. and HUXMAN M., Eur. Microsc. Anal. 3 (1992) 21-24.

[7] JEANGUILLAUME C. and COLLIEX C., Ultramicroscopy 28 (1989) 252-257.

[8] WILLIAMS D.B. and HUNT J.A., in Proc. Xth EUREM (Granada) Vol. 1, A. Rios, J.M. Arias, L. Megias-Megias, A. Lopez-Galindo Eds. (Secretariado de Publicaciones de la Universitad de Granada, 1992) 243-247.

[9] BALOSSIER G., THOMAS X., MICHEL J., WAGNER D., BONHOMME P., PUCHELLE E., PLOTON D., BONHOMME A. and PINON J.-M., Microsc. Microanal Microstruct. 2 (1991) 531-546.

[10] LEAPMAN R.D. and ANDREWS S.B., Microsc. Microanal Microstruct. 2 (1991) 387-394.

[11] REIMER L., Adv. Electron. Electron Opt. 81 (1991) 43-126.

[12] CASTAING R. and HENRY L., C.R. Acad. Sci. Paris B255 (1962) 76-78.

[13] SENOUSSI S., HENRY L. and CASTAING R., J. Microscopie 11 (1971) 19-28.

[14] SHUMAN H., CHANG C.F., BUHLE E.L. and SOMLYO A.P., Ann. N.Y. Acad. Sci. 483 (1986) 295-309.

[15] REIMER L., FROMM I. and RENNEKAMP R., Uttramicroscopy 24 (1988) 339-354.

[16] HUNT J.A. and WILLIAMS D.B., Ultramicroscopy 38 (1991) 47-73.

[17] EGERTON R.F., In Electron Loss Spectroscopy in the Electron Microscope (Plenum Press, New York 1986).

[18] LEAPMAN R.D. and HUNT J.A., Microscopy: The Key Research Tool 22 (1992) 39-51. 